\title{
Attraverso Deleuze e Simondon: ONTOGENeSi, PROCESSO E RELAZIONE
}

\section{Abstract}

Obiettivo del presente contributo è tracciare una cartografia della nozione di individuazione, nella sua relazionalità con la dimensione del pre-individuale, lavorando tra il pensiero di Gilles Deleuze e Gilbert Simondon, attraverso un percorso di ripensamento dell'idea di umanità. Si vorrebbe cioè mostrare come sia possibile risemantizzare la vexata quaestio della "Fine dell'Uomo", rileggendola piuttosto come un problema di con-fini e di margini, sempre attivi e divenienti. Al fine di mettere in luce questa costitutiva interrelazione fra il campo del pre-personale e dei processi di individuazione, si prenderanno in analisi i concetti di differenza, affettività e di "comunismo ontologico".

The aim of this work is to provide a cartography of the notion of individuation, in its relations with the dimension of the pre-individual, working between Gilles Deleuze and Gilbert Simondon, through a path of "fraying" of the human. Ultimately, we would like to show how it is possible to re-semantize the vexata quaestio of the "End of Man", re-reading it as a problem of borders and multiple layers, always active and in becoming. In order to highlight this constitutive interrelation between the field of the pre-personal and the processes of individuation, we will analyze the concepts of difference, affectivity and "ontological communism".

\section{INTRODUZIONE}

Nel presente articolo si vuole mostrare la possibilità di definire una filosofia che prenda seriamente in considerazione l'ipotesi teoretica di un "mondo-senza- di- noi", ${ }^{1}$ mettendo tuttavia in rilievo l'idea che il Fuori, da intendersi come energetica pre-individuale e pre-personale, si configuri come un'eccedenza che si contro-effettua prepotentemente $\mathrm{e}$ in fondo paradossalmente proprio all'interno della nostra individualità. Il soggetto in questa prospettiva si configura come una rete, un "fuoco lineare"2 che continua a individuarsi, come uno snodo e uno svincolo di molteplicità polivoche che si assemblano e agglutinano, sullo sfondo immanente e genetico di un campo pre-individuale a cui sempre siamo rinviati. In maniera corrispettiva il pre-individuale va concepito come una figura o immagine del pensiero, in quanto materia plastica che ci attraversa e a cui dobbiamo rivolgerci per comprendere al meglio la nostra fluidità e apertura sistemica.

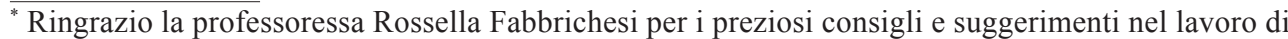
stesura della tesi di laurea magistrale, di cui l'articolo è una rielaborazione.

1 THACKeR 2018.

2 Deleuze 2004, p. 31.
} 
In definitiva si tenterà di mostrare come questa zona e territorialità topologica pre-individuale sia una dimensione ineliminabile e discriminante per poter concepire e pensare in profondità i concetti di relazione e processualità: è infatti solo a partire da una riflessione sull'essere pre-individuale che è possibile dare corpo a un'ontologia da intendersi come campo aperto di relazioni.

I concetti presi in analisi in questo elaborato si configurano tutti come modi della relazione: differenza, disparazione, trasduzione, allagmatica, strutturazione, individuazione e affettività sono cioè gli effetti di superficie di un fondo in-formale e relazionale.

In questa prospettiva e riflettendo su questi temi, si interrogherà in un comune "divenire-nel-mezzo" il pensiero di Gilles Deleuze e Gilbert Simondon, volendo istituire un terreno di alleanza fra i due autori, più che una mediazione dialettica o dialogica, dando spazio a una zona di indiscernibilità fra i due poli della relazione, ad un territorio dove non è più rilevante e preponderante stabilire a chi appartenga una determinata concettualità, ma mettere piuttosto in funzione e in movimento il pensiero, in risposta a nodi problematici. È lo stesso Deleuze a "utilizzare" Simondon, creando un terreno di indistinzione e liminarità con il suo pensiero. I riferimenti espliciti all'autore sono infatti molto pochi, e, fatta eccezione per i passaggi di Logica del Senso, ${ }^{3}$ di Differenza e Ripetizione, ${ }^{4}$ e dell'Isola deserta e altri scritti, ${ }^{5}$ dove Simondon è citato in maniera puntuale, si tratta per lo più di menzioni tangenziali presenti in nota. ${ }^{6}$

Quello che è più rilevante in definitiva per Deleuze è lavorare con e non sui concetti di Simondon, facendoli fluire in maniera impersonale e anonima all'interno della sua stessa riflessione.

\section{ESISTERE È DIFFERIRE: PER UN'ENERGETICA DELL'ESSERE}

Nel paragrafo che segue si vorrebbe tentare di proporre un dialogo fra il concetto simondoniano di disparazione e la nozione di differenza deleuziana. Una delle più interessanti e forse trascurate fonti della riflessione deleuziana attorno al concetto di differenza risiede nella metafisica monadologica di Gabriel Tarde. ${ }^{7}$ Per Tarde il reale è composto da «un tesoro di differenze impreviste». ${ }^{8}$ A suo avviso le differenze hanno un carattere intensivo, pragmatico e topologico più che estensivo ed essenziale, così come il differire non si presenta tanto come opposizione quanto come posizione affermativa e interna. Lungi dall'abolire i confini fra i diversi "domini" della realtà, la nozione di differenza non fa che «irridurli» (termine di Bruno Latour), ${ }^{9}$ ovvero inde-

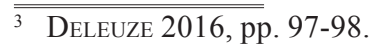

4 Deleuze 2015a; i riferimenti a Simondon sono presenti soprattutto nei capitoli Differenza in sé, L'immagine del pensiero e Sintesi ideale della differenza.

5 Deleuze 2007; cfr. nella fattispecie il saggio dedicato al pensiero di Simondon L'individuo e la sua genesi fisico-biologica.

6 Cfr. Deleuze 2010, p. 20.

7 TARDE 2013.

8 Ivi, p. 116.

9 LATOUR 1984. 
finirli, moltiplicandoli e rendendoli porosi, senza mai reificarli. Per irriduzione si intende cioè un movimento opposto ai riduzionismi, che tendono ad ingabbiare i fenomeni in un unico orizzonte teorico, livellando le differenze pragmatiche tra gli enti. Per Deleuze, molto tardianamente: «la differenza è dietro ogni cosa, ma dietro la differenza non c'è nulla», ${ }^{10}$ di rimbalzo le nozioni di identità, di contraddizione, di negativo e la corrispettiva nozione epistemica di rappresentazione non sono che "effetti ottici", immagini appiattite e rovesciate della differenza, modi del differire, che si configurano unicamente come un minimo, un punto zero di differenziazione. Mentre la contraddizione si risolve, in un processo di mediazione, la differenza, prolifera, va differendo. La differenza è un processo, un farsi: si fa la differenza. Al contrario il negativo porta con sé l'illusione della rappresentazione, della somiglianza, dell'analogia e dell'immagine, lasciando spazio a opposizioni categoriche e radicali. In ultima analisi per Deleuze il negativo di matrice hegeliana non cattura il fenomeno della differenza, ma ne carpisce soltanto il fantasma o l'epifenomeno: tutta la Fenomenologia $\grave{e}$ a suo avviso è in questo senso un'epifenomenologia.

Il fulcro del pensiero deleuziano sta nel non aderire a irrigidimenti duali ed escludenti, ma nell' includere piuttosto l'identità come un punto minimo della differenza. Non si nega in maniera assoluta l'esistenza di punti di agglutinazione sul tessuto reticolare del piano di immanenza: l'identità e il negativo continuano a sussistere come pieghe, punti limite di solidificazione di quell'Uno che è già da sempre plurale. Il ruolo della negazione non viene cioè negato, quanto piuttosto riorientato e scompaginato dall'interno. Centrale a proposito il concetto di equivoco: ${ }^{11}$ la filosofia deleuziana procede per soglie e paradossi, operando sintesi che non ha nulla di dialettico, ma che, in quanto sintesi disgiuntive si offrono come un modo relazionale nella distanza, tenendo insieme dimensioni incompatibili e disparate (come il negativo e la differenza).

I concetti simondoniani di disparazione, metastabilità, sovrassaturazione e translettica, (meglio esplicitati in seguito), colti nel loro rapporto inscindibile con il tema dell'individuazione intesa come divenire dell'essere costituiscono uno snodo teoretico presso il quale è discriminante soggiornare, se si vuole pensare in profondità e con serietà il problema differenza.

Simondon muta il termine disparazione dalla psico-fisiologia della percezione visiva, conferendole una piegatura filosofica nonché specificatamente ontologica. La disparazione binoculare in fisiologia si identifica come stato tensivo che si offre nella visione, fra l'occhio destro e l'occhio sinistro: a causa di una differenza nel parallasse, le immagini bidimensionali che giungono a ciascuna retina risultano sfasate, disparate, asimmetriche e non concordanti. Per risolvere (concetto fondamentale di cui Simondon farà un punto cardine della sua riflessione) questo stato di disparazione, il cervello umano produce un nuovo tipo di assiomatica, dando luogo alla visione tridimensionale, coerente e unitaria. La disparazione binoculare contiene in nuce il significato più profondo dei passaggi che Simondon applica in maniera trasposta alla sua

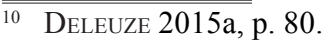

11 Viveiros de CASTRO 2004.
} 
ontologia: uno stato energetico di sfasamento, uno stato oggettivamente problematico e una risoluzione come amplificazione aperta che si offre a partire da uno stato problematico. ${ }^{12}$ Per l'autore francese il regime per eccellenza che si trova in uno stato di tensione disparante coincide con la sfera dell'essere:

l'essere non è mai uno, esso è più di uno [...] è più ricco della coerenza con sé, eccede i propri limiti, è metastabile, in espansione a partire da sé stesso; è trattenuto, teso, sovrapposto a sé stesso. L'essere non si riduce a ciò che è; è addensato in sé stesso, potenzializzato. Esiste come essere, ma anche come energia. ${ }^{13}$

Per sistema metastabile Simondon intende un sistema né stabile né instabile, un divenire-nel-mezzo fra staticità e squilibrio assoluto, uno stato energetico di equilibrio che si conserva fintantoché non viene fornito allo stesso un quantitativo di forze che ne perturbi l'omeostasi, una dimensione ricca di potenziali che agiranno quando sorgerà uno stato oggettivamente problematico.

Tramite il concetto di sovrassaturazione si chiarisce ulteriormente la nozione di metastabilità che di riflesso delinea a sua volta quella di disparazione. In chimica una soluzione sovrassatura è una soluzione in equilibrio instabile che contiene soluto in eccesso rispetto a quello che può contenere al suo livello di equilibrio termodinamico, così che, introducendo un piccolo frammento di soluto, essa va incontro all'improvvisa formazione di un precipitato.

Ancora una volta uno stato fisico-chimico diviene rappresentazione esemplare e prototipica di una triade fondamentale in Simondon: a uno stato di tensione energetica e di sovrassaturazione a livello dell'essere più che unitario (1) e all'insorgere di uno stato oggettivamente problematico all'interno del campo dell'essere (2), corrisponde una risoluzione dello stato problematico tramite il processo di individuazione dell'essere che si offre come divenire (3). L'essere è sovrabbondante, più che unitario, in movimento e relazionale, elude l'identità, il principio del terzo escluso nonché il principio di non contraddizione, offrendosi come plurale, doppio, sfasato. L'energetica differenziale è mossa da uno stato iniziale di tensione e di disparazione problematica al di sopra dell'unità. Essere è dunque $\delta v ́ v \alpha \mu \iota$, dinamogenesi di potenze differenziali. Il rapporto "sostanza" / "attributi" è una vexata quaestio che di sovente si traduce in una distanza radicale e ineliminabile fra gli stessi. Essere invece per Simondon è sinonimo di eterogenesi del divenire come individuazione (che si configura come un modo d'essere). Il divenire in questo senso non è una cornice dell'essere, ma una sua dimensione: «l'individuazione non è il risultato del divenire, né qualcosa che si produce nel divenire, ma il divenire stesso in quanto esso è divenire dell'essere». ${ }^{14}$

Molto similmente a Deleuze che invita a pensare il concetto di affermazione come proliferazione delle differenze, Simondon propone al lettore di concepire la disparazione come uno stato di tensione energetica conflittuale e agonistica, senza rifarsi an-

\footnotetext{
12 Si ricorda che Simondon oltre alle note pagine dell'Individuazione psichica e collettiva dedicò un intero corso di Psicologia generale al concetto di problematicità (Cfr. SIMONDON 2018).

13 Simondon 2001, p. 219.

14 Ivi, p. 28.
} 
cora una volta all'ipotesi di una contraddittorietà radicale, negativa e oppositiva. Egli prende in analisi e sottopone a critica tanto una forma di dialettica soggettiva ed epistemologica che pone il movimento dialettico nel soggetto, tanto una forma di dialettica oggettivo-reale che attribuisce il mutamento all'oggetto, proponendo invece quella che nelle pagine della sua opera viene definita come una translettica delle situazioni in cui soggetto e oggetto si danno simultaneamente: «la dialettica non è né dell'oggetto né del soggetto. Essa è della situazione, la quale costituisce una certa relazione mista, più originaria del soggetto e dell'oggetto. Essa è la sorgente della genesi. Oggetto e soggetto sono termini estremi». ${ }^{15}$

Dialettica è translettica, figura del paradosso di un'incompatibilità non oppositiva, punto di scarto, limite differenziale, indiscernibilità di eterogenei.

Deleuze, nella fattispecie in Differenza e Ripetizione, ma anche in Logica del senso e in un breve saggio dell'Isola deserta e altri scritti ha ben presente la riflessione ontologica simondoniana e, seppur non sempre in maniera esplicita, il suo debito nei confronti dell'autore nella sua concezione della differenza - che porta con sé una determinata e specifica teoria dell'individuazione e della nozione di problematicità - è inequivocabile. Da Simondon Deleuze mutua dunque l'idea di un essere da sempre in movimento, dinamico, energetico, in tensione disparante e non dialettica. Egli riprende l'idea simondoniana di un fondo oscuro recalcitrate l'individuazione, tingendolo dei connotati specifici del suo concetto di immanentismo, facendo riferimento allo stato di disprazione simondoniana come a una situazione di "molteplicità informale", come una profondità originale in uno stato di com-plicatio, letteralmente di piegatura accavallata di molteplicità. Il problema diventa il motore che sostituisce il vuoto scavato dallo spettro della negatività. Ed è alla nozione di problematicità che Deleuze dedica molte pagine di Differenza e Ripetizione, nella misura in cui il problema risiede al cuore del concetto di differenza, la abita, la fa funzionare, la fa in altri termini differire. Si può dire che il problema " $f a$ la differenza". Problema è ciò che muove l'ontogenesi (ovvero il processo di individuazione, da intendersi come divenire dell'essere). Secondo Deleuze, molto simondonianamente, il problema ha un carattere affettivo, nonché una dimora nello spazio logico-ontologico dell'evento. Egli scrive, nel suo testo dedicato allo strutturalismo: «eppure questo vuoto non è un non-essere; o almeno questo non-essere non è l'essere del negativo, è l'essere positivo del "problematico", l'essere oggettivo di un problema ${ }^{16}$ e di una domanda». ${ }^{17}$

Occorre sottolineare che tanto per Deleuze quanto per Simondon l'ontogenesi non risulta messa in movimento da problemi che si offrono come l'ombra di soluzioni preesistenti, date in forma proposizionale e ascritte all'ambito del vero e del falso, così come il concetto di riposta, lungi dall'implicare riferimenti antropomorfici, proposizionali e logocentrici, indica piuttosto uno stato oggettivo dell'essere.

\footnotetext{
15 SimONDON 2006.

16 Enfasi mia.

17 Deleuze 2015b, p. 57.
} 
Avendo analizzato da una parte il concetto di differenza deleuziana e dall'altra quello di disparazione simondoniana, notando le profonde influenze e suggestioni che Simondon esercita sul pensiero di Deleuze, resta da chiedersi se ci siano dei punti di fondamentale collisione teoretica fra $\mathrm{i}$ due pensatori. Per chiarire questo punto è necessario riferirsi al concetto di metodo trasduttivo che delinea in Simondon un'operazione biologica, fisica, mentale o sociale in base alla quale un'attività si propaga posto per posto (in progress), secondo una struttura reticolare amplificante, in modo costantemente variabile. L'esempio paradigmatico a cui Simondon fa riferimento sono i cristalli, nei quali un piccolo germe si estende in tutte le direzioni, generando una "presa di forma", ovvero la genesi di una struttura. A una prima lettura una tale operazione può suscitare un'obiezione legittima: come si fa a parlare di una filosofia delle relazioni nella misura in cui una forma, che sembra in qualche modo già formata, struttura un campo? Sembrerebbe in altre parole che il problema della genesi (seppur relazionale nel momento del suo strutturar-si) non possa slegarsi da un inizio assoluto, da un punto alfa da cui tutto si spieghi. Credo tuttavia che queste presunte derive teleologico-finaliste possano essere fugate tramite una lettura più attenta delle pagine simondoniane. Lo scoglio risiede cioè nel poter pensare a un processo ontogenetico-genealogico senza introdurre la figura di un inizio inteso in senso assoluto. Tra le pagine della filosofia simondoniana è contenuto lo scioglimento di questo nodo teoretico, nella misura in cui egli fa riferimento ad un "nascere", "apparire", "insorgere", della forma, del germe strutturale, che procede poi a un'operazione reticolare di "presa di forma": la forma, l'individuazione deve cioè formarsi a sua volta; essa non si configura come un termine, quanto piuttosto come una risposta ad un problema che emerge nello stato di metastabilità dell'essere sovrassaturo.

La forma non è un principio, un'origo primordiale da cui tutto a cascata si produce, strutturandosi, così come l'individuazione non mette in opera un principio già dato (la forma), ma lo crea. Si dà cioè un ripetersi aperto di questo semplice pattern:

\section{Stato metastabile dell'essere}

2. Emergere di uno "stato oggettivamente problematico" nel campo metastabile dell'essere

3. Risposta a uno stato oggettivamente problematico (divenire-forma dell'individuazione)

4. La forma formata struttura a sua volta il campo in un processo di amplificazione reticolare

5. Nuovo stato di sovrassaturazione metastabile

In questo senso l'individuazione nel suo formar-si non ha un principio, ma è relazionale, aperta e sempre accadente. Simondon prende le distanze da filosofie moniste dell'unità, muovendosi piuttosto nell'orizzonte teorico di un'ontologia della sovrabbondanza. L'essere si configura cioè nietzschenamente come una forza dinamitarda in esplosione $\mathrm{e}$ frattura energetica più che unitaria. L'autore francese sottopone a critica il concetto di unità nella misura in cui esso è troppo statico e stabile e non riesce a mettere in moto un processo 
di genesi individuativa, non spiegando le ragioni della polivocità dell'essere. Si può usare dunque Simondon per fare forse un passo oltre il monismo unitario senza rinunciare alla figura dell'immanenza, mettendo in luce quello che in fondo Deleuze presuppone senza tematizzare, ovvero una teoria della processualità. Forse questo aspetto rimane in Deleuze un po' in ombra e la filosofia di Simondon consente, per usare un'espressione nietzschiana, "di scagliare oltre la lancia" rispetto al monismo pluralista deleuziano. Resta tuttavia problematico abbandonare i "vantaggi" che una filosofia monista può offrire, difficile e aporetico pensare a una processualità più-che-unitaria del tutto immanente. La sfida è quella di tenere insieme l'essere "in esplosione" simondoniano all'immanenza di matrice deleuziana, senza rinunciare a una filosofia del processo. A mio avviso, infatti, il prezzo di una filosofia del processo non risiede tanto nella discrasia che viene a crearsi fra relazionalità genetica e immanentismo, quanto fra la prima e il concetto di monismo. La filosofia simondoniana chiede cioè di mettere da canto il monismo, senza rinunciare all'univocità immanente dell'essere che diviene: il divenire è un modo d'essere, una sua manifestazione effettuale che non sottende alcuna forza noumenica; è proprio nella sua manifestatitvità e non altrove che si può cogliere l'essere nel suo disparar-si. L'essere agisce come divenire, si effettua come tale in una processualità immanente, "una genesi senza inizio", già da sempre iniziata attraverso il pattern (stato metastabile-problema-risoluzione- e...e...e) in cui la potenza di generare effetti domina sul che cos'è.

\section{DAL PRINCIPIO DI INDIVIDUAZIONE A UN'INDIVIDUAZIONE SENZA PRINCIPIO}

Le prime pagine dell'individuazione psichica e collettiva sono dedicate al programmatico sovvertimento del paradigma classico in base al quale un individuo $\square$ in una certa misura già dato e precostituito in forma embrionale e del tutto potenziale $\square$, risulta passibile di un successivo processo di individuazione. Secondo questa visione, sottoposta a critica serrata da Simondon, un termine o principio di individuazione, seppur in maniera opaca e del tutto incipiente, prelude, prefigura e fa segno al darsi posteriore di un'entità individuata.

A suo avviso una presupposizione comune e condivisa inficia le argomentazioni filosofiche di una tale visione che si riverbera tanto nel sostanzialismo atomistico (di Leucippo, Democrito, Epicuro e Lucrezio nella fattispecie) quanto nell'ilemorfismo di matrice aristotelica; esse non sono che due modi eterogenei di fare lo stesso errore, ovvero quello di intendere il principio che guida l'individuazione come un qualcosa che contiene agglutinato in sé, come una premonizione, il suo sviluppo posteriore. Il principio di individuazione risulta in questo senso già caratterizzato dal modo d'essere dell'individuo; per Simondon in altri termini nella ricerca del principio di individuazione si postula erroneamente che l'individuazione abbia un principio.

Ontogenesi oltre che essere una definizione appartenente al vocabolario biologico è innanzitutto per Simondon un concetto ontologico che non concerne isolatamente l'individuo, quanto piuttosto una tensione processuale che attraversa la sfera più globale dell'essere. L'essere non è un concetto assoluto e astratto, ma lo stesso divenire 
processuale ed ontogenetico dell'individuazione, sempre suscettibile di riconfigurazioni e risemantizzazioni. In questo senso l'individuazione non è pensabile se non come individuazione dell'essere e l'essere non è concepibile se non come processo genetico-individuativo: non è la sola individuazione ad essere processuale, ma l'intera genetica dell'essere a presentarsi come diveniente. Ontologia è dunque sinonimo di ontogenesi genetico-individuativa.

Simondon dà corpo a una teoria allagmatica (dal greco $\grave{\alpha} \lambda \lambda \alpha \gamma \mu$ ó, ovvero scambio), di complementarietà e convertibilità ontologica tra operazione e struttura, facendo implodere dall'interno la coppia dicotomica tradizionale di materia e forma e rendendo piuttosto il loro rapporto un continuum di relazionalità aperta.

In Simondon in questo senso la forma è da assimilare sempre a un "divenir-forma", mentre la materia a un "materializzar-si" a opera di una forma. L'autore francese propone uno strutturalismo sui generis, molto più post-strutturale che classico, dove una processualità operante (ovvero la forma), generatasi in risposta a un problema che si pone in seno all'essere metastabile, struttura un ricettore. L'essere "in-forme" si configura come un campo di tensioni intensive molecolari, dove il processo di individuazione non è che un'emergenza, un punto di agglutinazione estensivo o molare. Il processo di individuazione (che si ricorda, va inteso nel suo ampio significato di divenire dell'essere), si gioca cioè nel rapporto fluido fra forma e materia.

Simondon, abbandonando l'idea di una forma che si imprime come uno stampo o calco circoscrivente su una materia grezza e informe, rende il rapporto tra i due poli al contrario una relazione di modulazione e di risonanza interna dove non c'è struttura strutturata che non sia stata a sua volta, precedentemente, un'operazione, e non c'è operazione che vicendevolmente non si converta in struttura.

È interessante soffermarsi su come Deleuze, nel suo breve testo dedicato allo strutturalismo, ${ }^{18}$ pieghi a sua volta il concetto di struttura tramite una torsione plastica e diveniente, avvicinandosi a quello che mi sembra l'intento simondoniano.

Così Deleuze, maneggiando il concetto di struttura, sia essa linguistica, letteraria, sociologica o antropologica, lo ridispone verso una via molto poco strutturalista, più attenta a ravvisare un movimento intensivo, operativo e aperto di strutturazione. Come in Simondon, anche in Deleuze, al centro della struttura abita una dinamica di problematicità irrevocabile e tensiva, «l'essere oggettivo di un problema e di una domanda». ${ }^{19}$

Il dinamismo della differenza, animato da stati oggettivamente problematici, non può in questo senso essere colto astrattamente in una struttura solida, rigida e offerta a priori, al di qua del suo offrirsi in effetti poietici, circostanziati e locali, ma, al contrario, è afferrabile solo nel suo movimento operativo e aperto di strutturazione. In altri termini avviene una risemantizzazione morfo-genetica del rapporto tradizionale tra materia e forma.

Deleuze, nel breve saggio contenuto nell'Isola deserta e altri scritti, Il metodo della drammatizzazione, chiarisce ulteriormente il doppio volto della differenza che fa

\footnotetext{
$\overline{\overline{18} \text { Deleuze 2004, p. }} 21$.

19 Ivi, p. 58.
} 
eco al darsi anfibio del costrutto concettuale simondoniano di materia/forma e dello speculare rapporto fra operazione e struttura, coniando il termine "different/zione". L'effetto della drammatizzazione (nella sua radice greca di $\delta \rho \alpha ́ \omega$, sinonimo di dinamismo plastico, di creatività poietica, di operatività pratica) consiste nella duplice effettualità della differenza che si configura tanto come differentiazione, nella misura in cui si collega al fondo virtuale pre-individuale, quanto come differenziazione in quanto attualizzazione individuativa. Il concetto di different/zione si riverbera nel rapporto

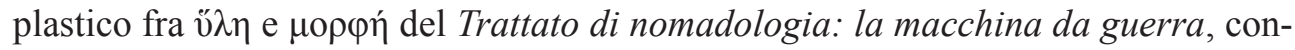
tenuto nel secondo tomo di Capitalismo e Schizofrenia (1972), dove Deleuze mette a tema in maniera magistrale una risemantizzazione radicale del rapporto fra materia e forma. A suo avviso infatti è centrale il concetto di disparità, in cui si può ravvisare un'eco simondoniana, da intendersi come un'energetica operazionale che inerisce a materiali-forze, fluide e dinamiche, più che alla coppia antipodale di materia e forma.

Il processo di formatura individuativa degli enti si configura cioè come un'operazione morfo-genetica, relazionale e dinamica, di materializzazione.

Nella riflessione deleuziana l'essenza di ogni individuo è in questo senso sempre vaga, in fieri e mai determinata in maniera ultima, presentandosi come costitutivamente anesatta.

Anche in Deleuze, così come in Simondon, i poli di virtualità e attualità, di individuazione e immanenza, di struttura e operazione si offrono in un rapporto di mutualità e scambio, di sintesi di disgiunti opachi e indiscernibili, sebbene ancora una volta sembri rimanere in ombra l'intento di sondare le profondità di un'analisi genetico-genealogica (pienamente presente in Simondon) del concetto di differenziazione come attualizzazione del virtuale. Come nota Michael Hardt: «Cos'è esattamente questo processo di recoupement $\mathrm{o}$ intersezione che collega la molteplicità attuale all'unità virtuale? Deleuze non tratta esaurientemente questo punto». ${ }^{20}$

OLTRE LA SCHIZO-ANALISI DELEUZIANA: IL “COMUNISMO ONTOLOGICO” DEL TRANSINDIVIDUALE

La riflessione di Deleuze si distanzia radicalmente da Simondon nella misura in cui l'intento decostruttivo rispetto al concetto di soggettività si unisce a un profondo spirito di spersonalizzazione. Lo stato etico per antonomasia coincide cioè per Deleuze con una completa, virulenta e dinamitarda esplosione dell'Io. Vibra fra le pagine del suo corpus la necessità di far fluire-altrimenti l'individuazione, dirigendola verso il flusso evenemenziale, singolare, immanente e pre-personale che coinvolge le flussioni e i movimenti del piano di immanenza. Per l'autore francese la nascita dell'identità che sopraggiunge a livello molare testimonia al contrario un atteggiamento nevrotico-paranoide di possesso del proprio Io; è il corpo anoressico, tossicomane, schizofrenico e dissociato a essere al contrario realmente immanente. Depersonalizzazione e derealizzazione allontanano il soggetto da sé stesso, appannandolo, rendendolo opaco e irreale, muovendolo verso l'anonimo: il corpo non è più una figura, ma uno sfondo

$\overline{20 \quad \text { HARDT } 2000, \text { p. } 59 .}$ 
di cui si perdono i confini, mentre la morte finisce per configurarsi come l'unico atto realmente immanente. L'immanenza in fondo è il modello della morte nella misura in cui essa si presenta come "un operatore metamorfico" per l'Io, che lo dissolve nell'Evento, rendendolo pienamente intensivo, liberando allo stesso tempo differenze libere, pre-personali e pre-individuali.

La riflessione di Deleuze è a mio avviso un punto di partenza ineliminabile nel panorama contemporaneo per avviare una riflessione non reificante sul concetto di soggetto: in Deleuze, infatti, l'ipotesi che sia sulla base di un fondo oscuro, artico e proteiforme che il processo di individuazione "prende forma" è il frutto di un'esigenza fondamentale, ovvero il voler sottolineare che è sempre in riferimento alla relazionalità del pre-personale che l'individuo può vivere e pensare. Questo processo di decostruzione del soggetto prende tuttavia troppo spesso nelle pagine dell'opera deleuziana una deriva eccessivamente radicale che scaturisce nel liquefarsi totale di una qualsiasi forma di emergenza soggettiva intesa come campo aperto, relazionale e reticolare di individuazione.

Credo che questa impasse possa essere superata rifacendosi alla riflessione di Simondon circa l'individuazione (psichica e collettiva nella fattispecie): l'azione transindividuale comune e affettiva presa in analisi da Simondon si presenta infatti come una valida alternativa alla depersonalizzazione schizofrenica che abita le pagine deleuziane, pur conservando l'intento di una filosofia immanente, che si riferisca a un tessuto di pre-individualità e pre-personalità. È cioè a mio avviso nel passaggio dal metodo schizo-analitico all'ipotesi di un "comunismo ontologico" che si gioca una via altra rispetto alla totale dissoluzione del soggetto.

Per Simondon l'individuo è imbarcato e imbricato nella relazionalità del pre-individuale, ne conserva un residuo non strutturato, libero e potenziale che recalcitra l'individuazione. L'individualità è doppia, anfibia, disparata e bimodale, tesa fra una zona

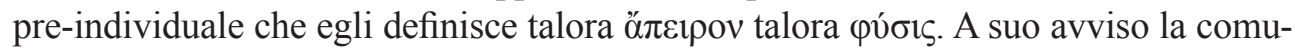
nicazione affettivo-emotiva e informazionale, la congiuntività e la relazionalità fra gli enti è possibile in virtù di questa presenza pre-individuale in ogni individuo. Il soggetto è sempre non sostanziale, relazionale e aperto. Intenderlo come circoscritto e perimetrale non è che una facilitazione e una concessione del e per il pensiero per poter assistere alla genesi e alla legittimazione narcisistica di un Sé monolitico e tabernacolare: «la sostanzializzazione del soggetto in quanto termine è una facilitazione che il pensiero si concede per poter assistere alla genesi e alla legittimazione di sé stesso». ${ }^{21}$ Occorre dunque sciogliere ogni sostanzializzazione assolutizzante, aprendosi al tessuto intrecciato della relazione, accettando che l'uomo è un essere doppio, costitutivamente anfibio e scisso fra una zona individuale di natura naturata ed una zona pre-individuale di natura naturans, che si configura come il pre-vitale, l'insoluto, il problematico e coincide con «una realtà che non va cercata nell'oggetto oggettivato né nel soggetto soggettivato, ma al confine tra l'individuo e ciò che rimane fuori di esso, secondo

$\overline{\overline{21 \quad \text { SimONDON 2001, }}}$ p. 189. 
una mediazione in bilico tra trascendenza ed immanenza». ${ }^{22}$ L'individuo per Simondon sperimenta questa eccedenza e dislocazione. È interessante notare come il concetto di sperimentazione rivesta un ruolo fondamentale anche nella riflessione di Deleuze: sperimentare è sinonimo di divenire-impersonali, di esperire una frattura che ci porta oltre noi stessi, in un senso non trascendente, ma topologico e geo-spaziale. L'immanenza è un luogo, una territorialità, una zona che ci precede ed eccede, ma che in una certa misura sempre ci appartiene. Sperimentare è ricongiungersi "estaticamente" (nel senso etimologico di uscita da sé) con il pre-personale che da sempre ci attraversa. L'immanenza non è un luogo altro trascendente, ma un altro modo di guardare le cose e il mondo, de-personalizzandosi e sfuggendo alle barriere narcisistiche e asmatiche del proprio Io. Per Simondon ancora una volta la questione è più sottile e meno unilaterale. In Deleuze, infatti, l'unica prospettiva plausibile risulta in ultima analisi lo sguardo da nessun luogo, immanente e singolare di un soggetto completamente dissociato e dissolto, mentre per Simondon la questione ruota attorno alla doppiezza, alla funzione-soglia che l'umano (e non umano) individuandosi "mette in scena". Se si intende infatti l'individuazione come un atto teatrale - ipotesi di Muriel Combes lettrice di Simondon $-{ }^{23}$ che va in scena su un doppio fondo, parimenti individuale e pre-individuale, si comprende la doppiezza costitutiva che caratterizza l'umano. Come si è già messo in luce, una base concettuale e teoretica comune si declina in maniera eterogenea fra i due pensatori: se per Simondon l'individuazione è infatti sempre anfibia e bimodale, per Deleuze l'unico sguardo possibile coincide in ultima analisi con un completo e definitivo processo di divenire-impercettibili, immanenti e impersonali. Simondon, in alcune delle pagine più liriche del testo L'individuazione psichica e collettiva mette a tema la nozione di transindividualità, collegandola al concetto di individuazione collettiva. È opportuno ricordare che i termini pre-individuale e transindividuale nonostante siano comunicanti e interrelati, non sono sovrapponibili e interscambiabili. Se per pre-individuale si intende infatti una carica di essere metastabile che attraversa l'individuazione, il transindividuale definisce invece il darsi a un tempo, simultaneo e reciproco dell'individuazione psichica e di quella collettiva. Per approdare al dominio del transindividuale che conduce all' "avventura" dell'individuazione collettiva occorre confrontarsi per Simondon con una situazione liminare, attraversare una solitudine paradossale, in quanto ricca di relazioni. Il ßá $\sigma \alpha v o \varsigma$ del transindividuale (ovvero la pietra di paragone con cui è necessario confrontarsi per accedere a tale dominio), coincide con un "rito di iniziazione" di spoliazione dalle funzioni sociali dei rapporti inter-individuali intesi come socialità grezza, come simulazione performativa e personologica, nel senso etimologico di mascheramento (e qui il riferimento di Simondon è Jung). Simondon adduce come esempio tipologico della prova del transindividuale l'episodio nietzschiano del funambolo abbandonato dalla folla. Quest'ultima lo aveva apprezzato solo per la sua funzione; lo abbandona quando, morendo, smette di esercitarla. Invece Zarathustra, sentendosi a lui affratellato, ne porta con sé il cadavere per dargli sepoltura, seguendo una fraternità assoluta e profonda, che eccede il dominio dell'individuale.

Ivi, p. 136.

23 Combes 2013. 
Aldi qua ßá бavos del transindividuale che si configura come un'operazione di dis-individuazione, (eterogenea rispetto a quella che prende corpo nel vissuto angoscioso in cui l'individuo resta problema per sé stesso), prende corpo una successiva individuazione, ovvero quella collettiva. Non è l'individuo, ma il pre-individuale insito nel soggetto a determinare un ulteriore processo di strutturazione che porta all'individuazione collettiva. Il pre-individuale dell'individuo non può realizzarsi né nel singolo soggetto né nella "comunità funzionale" inter-individuale, ma riesce a "prendere forma" solo nella misura in cui il soggetto mette in comune ciò che eccede i propri confini e limiti. Per Simondon chiudersi monoliticamente come un'entità insulare porta alla patogenesi: è necessario aprirsi al comune, alle zone di pre-individuale che ci attraversano, condividendo i potenziali energetici e dinamici che recalcitrano l'individuazione. Occorre ricordare che per l'autore francese il sociale non è un $\tau \hat{\lambda} \lambda \mathrm{s}$, così come l'uomo non è determinato essenzial-

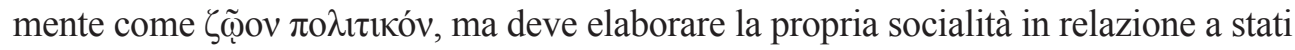
oggettivamente problematici. Il collettivo è in questo senso un processo che ha sempre da accadere. Per Simondon la vita del collettivo è la vita comune del significato, il mondo psico-sociale del transindividuale, "la verità pubblica" che scorre tra gli individui; c'è un corpo e una mente collettiva, un corpo sfrangiato e una mente estesa dove il pre-individuale residuo si individua, strutturandosi: il collettivo è corpo comune e Commens, ovvero mente condivisa e allargata. Come nota Rossella Fabbrichesi a proposito del tema del comune: «non si tratta di con-sentire, ma della costruzione di un corpo allargato, di una comunità complessa, di una mente estesa [...] Ogni individuo deve dunque rinunciare alla propria individualità e particolarità, per connettersi al Sé collettivo». ${ }^{24}$ Alla depersonalizzazione deleuziana - che in fondo è uno stato di apertura paradossalmente solipsistica - si sostituisce in Simondon una concezione relazionale ed aperta dell'individuazione, in cui l'Altro, inteso come Fuori, sia esso umano o non umano, ha un ruolo discriminante nella produzione di un' individuazione collettanea o collettiva (Paolo Godani definisce suggestivamente quest' apertura come "comunismo ontologico"). ${ }^{25}$

\section{IL CONCETTO DI AFFETTO, TRA PRE-INDIVIDUALITÀ E BIOSEMIOTICA}

Tra Deleuze e Simondon, senza negare in toto un ruolo al linguaggio, al pensiero, alle cognizioni (e così via) nella relazionalità umana, ma considerandoli come uno fra gli altri modi di comunicazione, espressione e modalità dello stare al mondo, si evidenzia l'esigenza di una "svolta affettiva".

Gli affetti in entrambi gli autori si presentano come dei passaggi, delle transizioni, degli scambi, mostrandosi come un dominio transindividuale, che elude i confini degli individui, attraversandoli e prendendo corpo ai loro bordi. Per Deleuze: «l'affetto non è un sentimento personale, e neanche un carattere, ma l'effettuazione di una potenza muta, che solleva e fa vacillare 1 'Io». ${ }^{26}$ In questo senso navighiamo nel mare degli affetti

\footnotetext{
$\overline{24}$ FABBRICHESI 2012, pp. 101-105.

25 Godani 2016, p. 33.

26 Deleuze - Guattari 2017, p. 299.
} 
e non siamo il timoniere di una nave in nostro controllo, quanto piuttosto gli spettatori di un'energia teatrale che va in scena e che ci precede, con cui possiamo solo fluire.

L'affetto si configura in ultima analisi come un indice "barometrico" del divenire, delle sue pressioni e forze, delle sue dinamiche, dei suoi movimenti e delle sue flussioni.

Anche la riflessione di Simondon sull'affettività è molto ampia, stratificata e composita, e ciò non sfugge a Deleuze che in un saggio dell'Isola deserta e altri scritti, dedicato a Simondon, ci ricorda in maniera tangenziale, ma incisiva, come a suo avviso l'autore sviluppi: «una teoria molto interessante dell'affettività». ${ }^{27} \mathrm{La}$ teoria degli affetti di Simondon si colloca entro una prospettiva ontogenetica, nella misura in cui la sua ontologia è mossa dalle cariche dinamogenetiche dell'essere e da una condizione di metastabilità problematica: è l'essere in primo luogo ad affettare e ad essere simultaneamente affetto dai movimenti che avvengono fra le sue pieghe; a cascata gli affetti colorano e caratterizzano il processo ontogenetico nella sua globalità, sprigionandosi anche nel divenire in quanto modo dell'essere.

Per Simondon è inoltre centrale il concetto di comunicazione: esso ha una carica affettiva e trasduce informazione, precedendo la vita individuata, (per poi sprigionarsi anche in tale dominio), essendo in primo luogo una dimensione dell'essere pre-individuale. Egli, proponendo un uso trasversale della nozione di informazione e segnale, si avvicina ai moderni studi di biosemiotica ${ }^{28}$ che partendo dalla semiotica di Peirce, sviluppano un'idea "immanente" e transpecifica del concetto di segno. È in primo luogo la vita, intesa come il fluire pre-individuale dell'essere che diviene, a essere in sé stessa semiotica, rinviante, aperta e relazionale.

La "semiotica" simondoniana intesa come produzione di informazione, di intermezzi, scambi e divenire-altro, coinvolge il mondo animale, il mondo vegetale, il mondo umano, il dominio dell'inorganico, dell'in-umano, e così via: tutto è informazione, comunicazione e transito, dal momento che è l'essere stesso a presentarsi come affettivo, comunicativo ed energetico.

\section{FINE O CON-FINI DELL'UOMO?}

Tra Simondon e Deleuze il tema della morte (e più in generale della morte dell'Uomo) è esplorato in maniera profonda e capillare. La "fine dell'Uomo" è un'invenzione o meglio, un'ossessione recente, si potrebbe dire, parafrasando Foucault.

In queste note conclusive si vuole tentare di mostrare come sia possibile (e forse auspicabile) risemantizzare la vexata quaestio della fine dell'umano rileggendola come un problema di con-fini, di "sfrangiamenti" multipli, sempre attivi e divenienti. Questo percorso di "smarginamento" dell'umano prende forma attraverso Deleuze e Simondon, tra i concetti di differenza, disparazione, allagmatica, immanenza, individuazione e affettività, e raggiunge il suo vertice nella riflessione degli autori

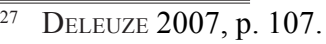

28 Romanini - FERNANDEZ 2014.
} 
sulla nozione di morte.

Deleuze fa riferimento all'idea che dopo la morte qualcosa resista allo scompaginamento. Vorrei provare a sostenere che dietro quello che sembra uno scacco all'immanentismo, si celi in realtà l'idea che effettivamente qualcosa passi attraverso la morte, in un senso topologico e transindividuale. Per Deleuze, infatti, la morte è una morte "senza fine" 29 e continua, un "Si muore" à la Blanchot, nella misura in cui non si muore in un istante puntuale, ma si continua a morire dal momento che si è vivi, così come si continua a vivere dal momento che si è morti. Se per il cartesianesimo la vita è tutta in unico luogo, mentre ogni altro luogo è un luogo morto, per Deleuze, lettore di Leibniz, vita e morte transitano e trascolorano l'una nell'altra, in un processo di "divenire-nel-mezzo", componendo limitrofie e smarginandosi l'una nell'altra. Allo stesso modo per Simondon, c'è qualcosa di “eterno" nell'individuo, che si reintegra e ricompone dopo la morte, secondo un regime affettivo. La morte non è un istante discreto e puntuale, ma un lento scivolare, costantemente riattivabile, degli effetti prodotti in vita. Finché gli a/effetti continuano cioè a strutturare relazioni transindividuali, sono attivi, dinamici, energetici. La morte in questo senso è una doppia soglia nella misura in cui si presenta in primo luogo come l'evento transindividuale per antonomasia, dove il pre-individuale ai bordi degli individui viene rievocato in una processualità aperta di véкvi $\alpha$ (concetto mutuato da Simondon dalla tradizione dell'antica Grecia, che delinea uno spazio relazionale di "evocazione affettiva" e rituale dei

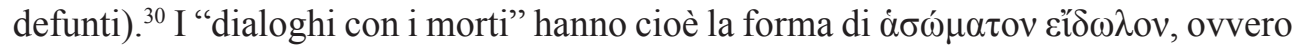
di immagini incorporee, transindividuali, singolari e immanenti, che rappresentano il nucleo più intimamente relazionale degli individui. A proposito è interessante notare come anche per Deleuze l'incorporeo non concerna né azioni fisiche né tanto meno spiritualità metafisiche, quanto piuttosto il tempo del puro divenire, inteso come zona di scambi e tramiti: l'incorporeo è l'Evento, l'effetto di superficie che transita fra i corpi e fra gli individui. In secondo luogo, la morte è intesa come uno sconfinamento liminare poiché ha i tratti di un trapasso continuo, sempre accadente e mai concluso in maniera recisa e puntuale.

La vita è cioè da sempre auto-immune, permeabile e plastica, costantemente riattivabile e coesistente con il Fuori. Da questo vertice osservativo, pensando al concetto di relazione, nella sua radicalità, intendendolo come soglia o limite, si comprende come il tema della fine o della morte dell'uomo sia in fondo un falso problema: la spiegazione genetica del processo che offre la filosofia simondoniana, esplicitando il tema della genesi delle relazioni del piano di immanenza, rimasto forse presupposto nella riflessione deleuziana, evidenzia infatti come ogni processo sia già da sempre iniziato, e dunque privo di un'origine absoluta, sciolta da qualsiasi legame e im-plicazione. Del resto, la genesi (individuativa, differenziale, affettiva etc.) è sempre accadente ed evenemenziale, mai delimitabile, circoscrivibile o perimetrabile. Essa è mossa dal suo dentro più intimo, da un Fuori problematico, energetico e tensivo. Per Simondon

\footnotetext{
Deleuze 2020, p. 30.

30 Simondon 2001, pp. 105-106.
} 
in questo senso l'immagine dell'uomo è radicalmente ripensata, smarginata e sfrangiata, ma mai considerabile conchiusa, delimitata o conclusa.

Pensare la fine dell'uomo significa in altre parole replicare il problema di un' $\alpha \rho \chi \eta ́$ metafisica, percorrendo controcorrente un fiume molte volte attraversato dalla filosofia, ovvero quello della metafisica. Più che di fine, dunque, è forse necessario parlare di con-fini, relazioni, limiti, soglie e passaggi umani, poiché, come nota Derrida nel saggio Fini dell'uomo: «il pensiero della fine dell'uomo è sempre già prescritto nella metafisica». ${ }^{31}$ La fine è una figura ambigua, doppia, la cui stessa etimologia rimanda a un legame fra un'idea teleologica e un compimento ultimo, definitivo, assoluto. Per Simondon non si dà fine poiché non c'è inizio: lo spazio della relazione ha piuttosto

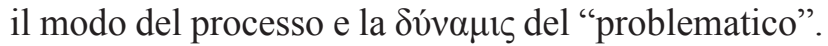

In definitiva, se Simondon, più di Deleuze, erode l'idea di un inizio e di una fine assoluta, spiegando il movimento e la relazione nella loro processualità ontogenetica e operante, Deleuze del resto, pur lasciando il tema della genesi in fondo presupposto, mette in scena e concretizza nei suoi testi il movimento, la relazione e la processualità in quella che Badiou definisce molto suggestivamente un' "avventura del concetto" o "una dimensione piratesca della filosofia". ${ }^{32}$ Lo stile deleuziano non è dunque un mero accessorio, ma un esercizio del pensiero a "fare il movimento", che trascina il linguaggio attraverso i concetti e la loro dinamicità.

Silvia Zanelli

Università degli Studi di Bergamo silviazanelli28@gmail.com

\section{RIFERIMENTI BIBLIOGRAFICI}

Combes 2013 : M. Combes, Gilbert Simondon and the Philosophy of the Transindividual, Cambridge (Mass.), MIT Press, 2013.

Deleuze 2004 : G. Deleuze, La piega, Leibniz e il Barocco, a cura di D. Tarizzo, Torino, Einaudi, 2004 (I ed. 1988).

Deleuze 2007 : G. Deleuze, L'isola deserta e altri scritti, a cura di D. Borca, Torino, Einaudi, 2007 (I ed. 2002).

Deleuze 2010 : G. Deleuze, Cinema, a cura di M. Bertolini, Milano-Udine, Mimesis, 2010 (I ed. 1983).

\footnotetext{
$\overline{\overline{31} \text { DERRIDA 1997, p. }} \cdot 107$.

32 BADIOU 2017, p. 46.
} 
Deleuze 2015 : G. Deleuze, Differenza e ripetizione, a cura di G. Guglielmi, Milano, Raffaello Cortina, 2015 (I ed. 1968).

Deleuze 2015b : G. Deleuze Lo strutturalismo, a cura di S. Paolini, Milano, SE, 2015 (i ed. 1976).

Deleuze 2016 : G. Deleuze, Logica del senso, a cura di M. De Stefanis, Milano, Feltrinelli, 2016 (I ed. 1969).

Deleuze - Guattari 2017 : G. Deleuze - F. Guattari, Mille Piani, a cura di P. Vignola, NapoliSalerno, Orthotes, 2017 (I ed.1980).

Deleuze 2020 : G. Deleuze, La soggettivazione [1985-1986], a cura di C. De Michele, Verona, Ombre Corte, 2020.

DerRida 1997 : J. Derrida, Margini della filosofia, a cura di M. Iofrida, Torino, Einaudi, 1997 (I ed. 1972).

FABBRICHESI 2012 : R. Fabbrichesi, In comune. Dal corpo proprio al corpo comunitario, Milano-Udine, Mimesis, 2012.

GodAni 2016 : P. Godani, La vita comune, Roma, DeriveApprodi, 2016.

Hardt 2000 : M. Hardt, Gilles Deleuze: un apprendistato in filosofia, a cura di G. De Michele, Milano, AChange, 2000 (I ed. 1993).

Latour 1984: B. Latour, Les microbes. Guerre et paix. Suivi de Irréductions, Paris, A.M. Métailié, 1984.

Romanini - Fernandez 2014: Peirce and biosemiotics. A Guess at the Riddle of Life, a cura di V. Romanini - E. Fernandez, Berlin, Springer, 2014.

Simondon 2001 : G. Simondon, L'individuazione psichica e collettiva, a cura di P. Virno, Roma, DeriveApprodi, 2001 (I ed. 1989).

Simondon 2004 : G. Simondon, Deux leçons sur l'animal et l'homme, Paris, Ellipses, 2004.

Simondon 2006 : G. Simondon, Per una nozione di situazione dialettica, «Il Protagora» 5, 5 (2006), pp. 105-119.

Simondon 2018 : Gilbert Simondon, La résolution des problems, Paris, PUF, 2018.

TARde 2013 : G. Tarde, Monadologia e sociologia, a cura di F. Domenicali, Verona, Ombre Corte, 2013 (I ed. 1895).

THACKer 2018 : E. Thacker, Tra le ceneri di questo pianeta, l'orrore della filosofia, la filosofia dell'errore, a cura di C. Kulesko, Roma, Nero, 2018 (I ed. 2011).

Viveiros de CASTro 2004 : E. Viveiros de Castro, Perspectival Anthropology and the Method of Controlled Equivocation, «Tipití: Journal of the Society for the Anthropology of Lowland South America» 2, 1 (2004), pp. 3-22 (https://digitalcommons.trinity.edu/tipiti/, ultimo accesso: 11 giugno 2021). 